\title{
Women with breast cancer were more satisfied with general practitioner care than with outpatient clinic care
}

Grunfeld E, Fitzpatrick R, Mant D, et al. Comparison of breast cancer patient satisfaction with follow-up in primary care versus specialist care: results from a randomized controlled trial. Br J Gen Pract 1999 Sep;49:705-10.

QUESTION: In women with breast cancer in remission, does the level of satisfaction with health service delivery differ when follow up is done in a primary care setting rather than in a specialist care hospital outpatient setting?

\section{Design}

Randomised \{allocation concealed*⿻千, unblinded,* controlled trial with 18 month follow up.

\section{Setting}

2 district general hospitals in the UK

\section{Patients}

296 women (mean age 61 y) who had breast cancer in remission and were receiving follow up care at 1 of 2 general hospitals. 266 women (90\%) and 256 women $(86 \%)$ completed questionnaires at the middle and end of the trial, respectively.

\section{Intervention}

Women were allocated to follow up care by their own general practitioner $(\mathrm{GP})(\mathrm{n}=148)$ or to usual follow up care from 1 of the 2 hospital outpatient clinics $(n=148)$.

\section{Main outcome measures}

Patient satisfaction was measured using a questionnaire developed by the UK College of Health. For analysis, the statements were grouped into 3 categories: those relating to service delivery, consultation, and continuity of care. Choices for response were "agree," "agree sometimes," "disagree," and "can't say." The first 2 were grouped together in the analysis.

\section{Main results}

Mid-trial results were reported because they were completed within 10 days of a follow up visit and did not differ from end of trial results. Analysis was by intention to treat. At mid-trial, women who received follow up care from their GP indicated greater satisfaction than did women who received routine follow up care in an outpatient clinic; statistically significant differences were seen between groups for responses to 9 of 12 statements $(p \leqslant 0.009)$. For all but 2 statements about the consultation, more GP group patients indicated improved satisfaction at mid-trial over that at baseline than did patients in the outpatient clinic group. Hospital outpatients showed greater satisfaction from baseline with only 1 statement about service delivery.

\section{Conclusion}

Women with breast cancer in remission had a higher level of satisfaction with health service delivery when follow up was done by their general practitioner than when it was done in a specialist care hospital setting.

*See glossary.

†Information from BMJ 1996;313:665-9.

\section{COMMENTARY}

The observation that patients with breast cancer in remission are more satisfied with follow up in a general practice setting than in a hospital specialist clinic is intriguing. However, as Grunfeld and colleagues recognise, satisfaction with care is an important outcome measure, particularly when it has been shown that medical outcomes are not affected. Although a previous report of this study suggests that this lack of clinical effect is the case, ${ }^{1}$ too few events for which early treatment is important (particularly local recurrences after lumpectomy) occurred to be confident that differences did not exist. Pending confirmation, the current report suggests an approach to follow up that can be considered in the future. For women who are willing to consider follow up in a general practice setting (one third were not) and for GPs who are willing to accept this responsibility (in an Ontario survey 10\% were reportedly unwilling), ${ }^{2}$ general practice is a possible option. However, whether sufficient unused general practice resources exist to meet this demand has not been addressed. Patients who have strong preferences for follow up in specialist settings, and for whom follow up in such settings would be most appropriate (eg, those with treatment related complications), will continue to exist. Furthermore, patient preferences and satisfaction with care may differ considerably in health care systems other than those of the British National Health Service. Additional research with longer follow up is needed to understand the effect of follow up on medical outcomes and to ensure that satisfaction with care in general practice, measured early after transfer of care to that setting, did not reflect response to a "new situation" and that it continues with prolonged follow up. Another study by Grunfeld and colleagues is currently being done, and results that may clarify some of these issues are expected in about 2 years. ${ }^{3}$ Satisfaction measured by other groups and in the health care systems of other countries should also be evaluated.

\section{Pamela J Goodwin, MD, MSc Mount Sinai Hospital Toronto, Ontario, Canada}

1 Grunfeld E, Mant D, Yudkin P, et al. Routine follow up of breast cancer in primary care: randomised trial. $B M J$ 1996;313:665-9

2 Worster A, Bass MJ, Wood ML. Willingness to follow breast cancer. Survey of family physicians. Can Fam Physician 1996;42:263-8.

3 Grunfeld E, Levine M, Coyle D, et al. A status report on a randomized trial of follow-up strategies for breast cancer. Canadian Breast Cancer Research Initiative: reasons for hope. Toronto, June 1999.
Sources of funding: Department of Health for England and Wales, Ballakermean School of the Isle of Man; Imperial Cancer Research Fund.

For correspondence: Dr E Grunfeld, Ottawa Regional Cancer Centre, Department of Medicine, University of Ottawa, 501 Smyth Road, Ottawa, Ontario K1H 8L6, Canada. Fax +1613725 6308 . 